\title{
Malignant pericardial mesothelioma: diagnostic clues in multimodality imaging
}

\author{
Dae-Young Kim ${ }^{1}$ - Young Nam Yoon ${ }^{1} \cdot$ Geu-Ru Hong ${ }^{1} \cdot$ Jong-Won $\mathrm{Ha}^{1} \cdot$ Chi Young Shim ${ }^{1}$
}

Received: 21 March 2020 / Accepted: 26 March 2020 / Published online: 6 April 2020

(c) Springer Nature B.V. 2020

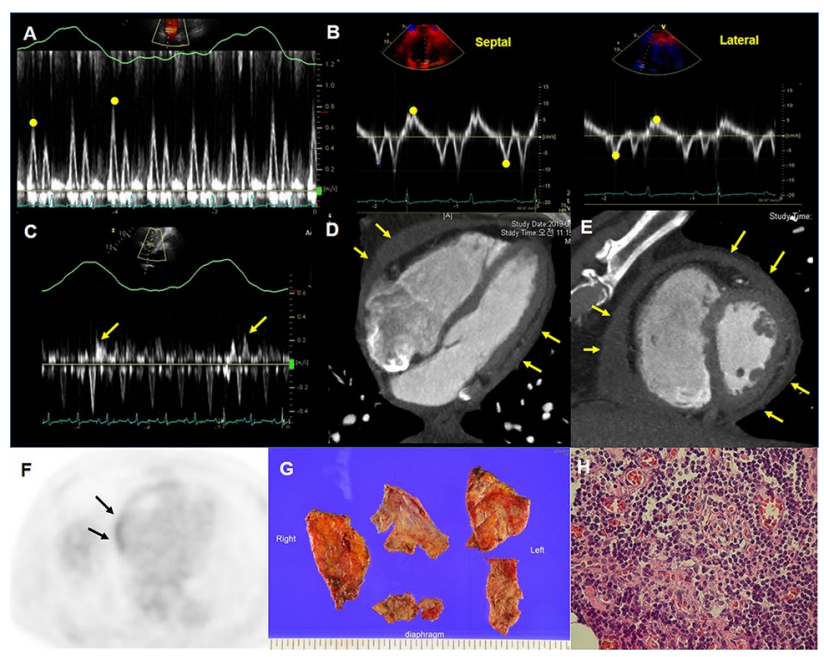

A 75-year-old man was presented with progressive dyspnea for 6 months. He denied a history of pulmonary tuberculosis and any systemic disease. His body temperature was $36.6^{\circ} \mathrm{C}$. On physical examination, his jugular vein was distended and pitting edema was noted on both legs. Chest radiography showed mild cardiomegaly, and his electrocardiogram revealed sinus rhythm without specific abnormalities. Laboratory findings showed elevated N-terminal pro B-type natriuretic peptide, hepatic enzymes but, normal ranged inflammatory markers and thyroid function. Echocardiography demonstrated thickened pericardium, paradoxical septal motion, and inferior vena cava plethora (See Supplementary data online, Videos S1-S4). Doppler evaluation showed respiratory variation of mitral early diastolic velocity, annulus reversus, and expiratory diastolic reversal of hepatic vein, compatible with constrictive physiology (Panels A-C). Computed tomography (CT) revealed diffuse pericardial thickening without significant calcification (Panels D and E). There was mild FDG uptake in

Electronic supplementary material The online version of this article (https://doi.org/10.1007/s10554-020-01829-1) contains supplementary material, which is available to authorized users. the area of thickened pericardium on positron emission tomography (PET) (Panel F). Because he had progressive symptoms despite medical treatment, pericardiectomy was performed. Thickened pericardium and severe adhesion were found and thereafter detaching and resection of pericardium from adjacent myocardium were performed (Panel G). Histopathology indicated malignant mesothelioma, epithelioid type (Panel H). The immunohistochemistry with podoplanin (D2-40), cytokeratin (CK) 5/6, CK (AE1/AE3) were positive, and Wilm's tumor gene (WT)-1 was weak positive, which were consistent with mesothelioma. This case displays the characteristics of malignant pericardial mesothelioma on multimodality imaging, which are constrictive physiology on echocardiography and a markedly thickened pericardium without pericardial calcification on CT, and little FDG uptake on PET.

Publisher's Note Springer Nature remains neutral with regard to jurisdictional claims in published maps and institutional affiliations.

Chi Young Shim

cysprs@yuhs.ac

1 Yonsei University College of Medicine, Seoul, Korea 